\title{
LPV Model for PV Cells and Fractional Control of DC/DC Converter for Photovoltaic Systems
}

\author{
Rubén Martínez, Yolanda Bolea and Antoni Grau \\ Automatic Control Dept. \\ Technical University of Catalonia \\ Barcelona, Spain. \\ Email: \{ruben.martinez.gonzalez,yolanda.bolea,antoni.grau\}@upc.edu
}

\author{
Herminio Martínez \\ Electronics Engineering Department \\ Technical University of Catalonia \\ Barcelona, Spain. \\ Email: herminio.martinez@upc.edu
}

\begin{abstract}
This paper deals with the fractional modelling of a DC-DC converter, suitable in solar-powered electrical generation systems, and the design of a fractional controller for the aforementioned switching converter. A new model for PV cells is proposed in order to obtain a linear equation for $\mathrm{V}$ I characteristic via scheduling dependence of temperature and irradiance. Due to the fractional nature of the ultracapacitors this kind of controller gives a suitable and good performance.
\end{abstract}

\section{INTRODUCTION}

Electricity production by renewable energy sources is actually promoted in many countries worldwide and is considered a strategic objective for the next years. Solar cells represent promising alternative that will likely initially supplement fossil fuel based energy supply, and eventually replace the fossil fuel energy sources as the availability of the latter declines.

However, photovoltaic (PV) generation systems, Figure 1, have two major problems which are related to low conversion efficiency of about 9 to $12 \%$ especially in low irradiation conditions and that the amount of electric power generated by PV arrays varies continuously with weather conditions. Therefore, considerable research is being carried out to increase the efficiency of the energy produced from PV systems [1], [2].

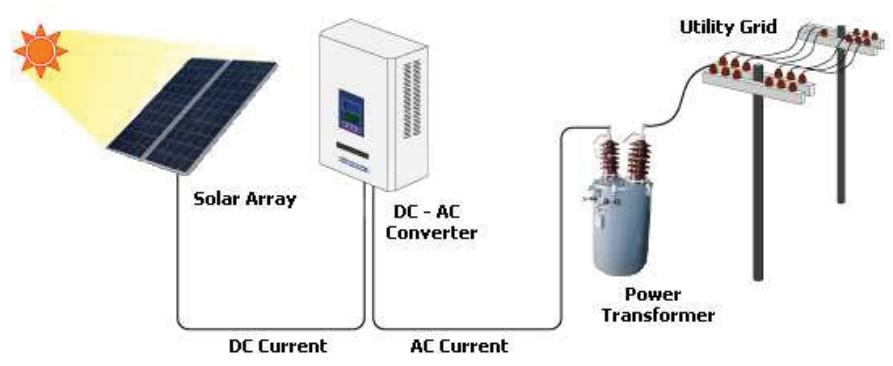

Fig. 1. Components of a typical on-grid PV system.

For any PV system, one option for increasing its output power is by tracking the maximum power point (MPP) of the PV system. The solar cell current-voltage (I-V) characteristic is nonlinear and varies with irradiation and temperature. However, there is a point on the I-V and power-voltage (P-V) curve, called as the MPP, in which at this point the PV system is said

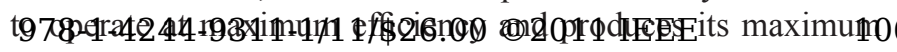
power output. The location of the MPP is not known but can be traced by using maximum power point tracking (MPPT) methods to maintain the PV array operating point at its MPP.

Usually, a DC-DC converter is used in PV systems as a controlled energy-transfer equipment between the main energy source (PV arrays) and a load or auxiliary energy storage system, recently based on ultracapacitors. Some advantages of ultracapacitors over more traditional energy storage devices include high power capability, long life, a wide thermal operating range, low weight, flexible packaging, and low maintenance [3].

The performance characteristics of ultracapacitors differ somewhat from those of conventional capacitors. The impedance of any real ultracapacitor can be easily reproduced in any frequency model equation by replacing every $j w$ expression with $(j w)^{\alpha}, 0<\alpha<1$, and where $\alpha=1$ represents an ideal capacitor with no frequency dependence [3]. Experimentally, the parameter $\alpha$ is not often smaller than 0.5 , the case of a Warburg impedance. A single value of $\alpha$ normally describes an electrochemical system over only a limited frequency range [4].

This non-ideality is a typical feature of electrochemical charging processes, and may be interpreted as resulting from a distribution in macroscopic path lengths (non-uniform active layer thickness) or a distribution in microscopic charge transfer rates, adsorption processes, or surface roughness [3]. For distributed parameter systems, it has been shown that fractional order calculus will play a role in its modeling and analysis. In general, fractional-order systems are useful to model various stable physical phenomena (commonly diffusive systems) with anomalous decay, say those that are not of exponential type.

In this work, we focus on the control of a converter based on ultracapacitors as an essential element in the optimal use of available energy in the PV arrays. A fractional control approach is motivated by the fractional nature that presents the model of the converter with ultracapacitors as accumulator. Furthermore, a linear parameter varying (LPV) algorithm is proposed in order to estimate the output voltage at MPP.

\section{A Survey of Fractional Calculus}

The idea of non-integer order derivates is as old as regular gralculus. Fractional calculus has been used for modelling different physical phenomena [5] and in control theory [6], 
[7], [8]. We can observe systems in the nature with fractional behaviour, but many of them with a very low fractionality [9].

The fractional integral operator is defined by [5]

$$
I_{t}^{\alpha} f(t)=\frac{1}{\Gamma(\alpha)} \int_{0}^{t}(t-\tau)^{\alpha-1} f(\tau) d \tau
$$

and we adopt the Caputo definition for fractional derivative of order $\alpha$ of any function $f(t)$ :

$$
\begin{gathered}
D^{\alpha} f(t)=I^{n-\alpha} D^{n} f(t) \triangleq \frac{1}{\Gamma(n-\alpha)} \int_{0}^{t} \frac{f^{(n)(\tau)}}{(t-\tau)^{\alpha-n+1}} d \tau \\
n-1<\alpha<n, \quad \alpha \in \mathbb{R}^{+}
\end{gathered}
$$

where the gamma function $\Gamma(\nu)$ is defined for $\nu>0$ as:

$$
\Gamma(\nu)=\int_{0}^{\infty} x^{\nu-1} e^{-x} d x
$$

\section{Modelling the Solar Cell}

Mathematical descriptions of the I-V characteristics of PV cells are available since many years and are derived from the physics of the p-n semiconductor junction.

The simplest equivalent circuit of a PV cell (Fig. 2) is a current source whose intensity is proportional to the incident radiation, in parallel to a diode with an ideality factor $n$, and a shunt resistance $R_{s h}$. This resistance represents the leakage current to the ground. The internal losses due to current flow and the connection between cells are modelled as a small serial resistance $R_{s}$.

Equation (4) is a general expression for the current produced by a real solar cell [11]:

$$
I=I_{s c}-I_{0}\left(e^{q\left(V-I R_{s}\right) / n k T}-1\right)-\frac{V+I R_{s}}{R_{s h}}
$$

where the short-circuit current $I_{s c}[a m p]$ and dark saturation current $I_{0}$ are given by rather complex expressions that depend on the solar cell structure, material properties, and the operating conditions. $G\left[\mathrm{~kW} / \mathrm{m}^{2}\right]$ (the solar irradiation), $k=1.3806 \times 10^{-23}[J / K]$ (the Boltzmann's constant), $q=1.6 \times 10^{-19}[C]$ (the electron charge), $T[K]$ (the temperature) , $1 \leq n \leq 2$ (the diode nonideality factor) and $N_{s}$ (the number of cells in series) are other parameters used to determinate Equation (4).

Other more accurate models are also available, such as a two-diode equivalent circuit (and the corresponding doubleexponential equation), which is particularly suited for polycrystalline cells [12]. A closed-form exact solution for Equation (4) (or for the double-exponential equation) for the unknown current $I$ is not available, hence numerical methods should be used to solve it.

An example of the current-voltage I-V characteristic of a typical silicon solar cell is plotted in Figure 3.

Equation (4) by itself does not lead to draw the I-V curve: the temperature dependence of the photo-current, the value of open-circuit voltage $\left(V_{o c}\right)$ and the short-circuit current $\left(I_{s} \mathbb{1} 070\right.$ are necessary to complete the model [13].

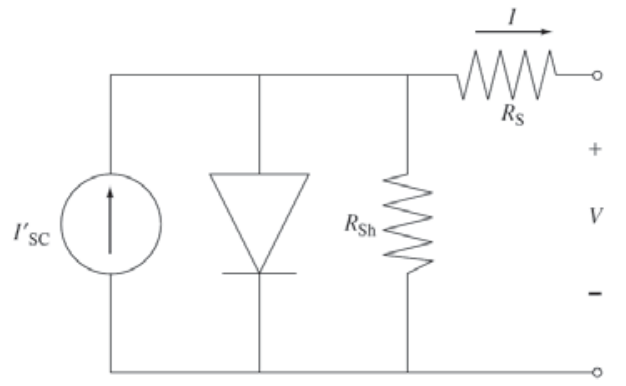

Fig. 2. Equivalent circuit of a PV cell.

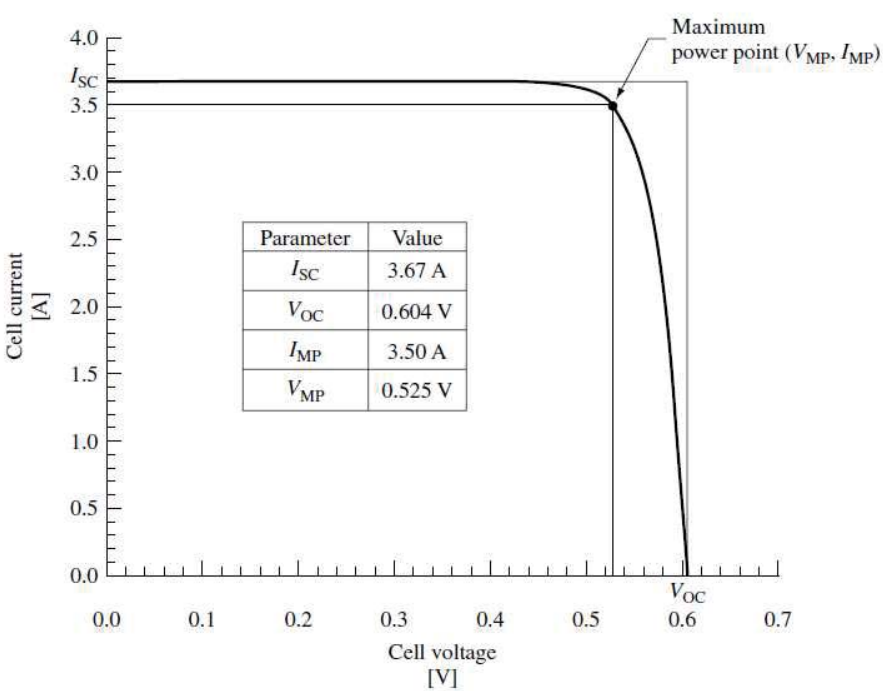

Fig. 3. Example of $I-V$ characteristic for the silicon solar cell.

Figure 4 is an example of the dependence of $V_{o c}$ and $I_{s c}$ versus light and temperature. However, manufacturers only provide the temperature dependence of $V_{o c}$ and $I_{s c}$, and irradiation dependece of $I_{s c}$ through the NOCT parameters and the electrical characteristics. In order to complete the PV cells model is necessary the value of $V_{o c}$ vs irradiation.

In this work a new PV cell model is proposed. The dependence of $V_{o c}$ and $I_{s c}$ vs irradiation (G) and temperature (T) as scheduling parameters are used into a linear parameter varying (LPV) model defined by:

$$
I=I_{s c}^{(G, T)}\left\{1-\lambda_{1}^{(G, T)} e^{q\left[V-V_{o c}^{(G, T)}\right] /\left[N_{s} n k T \lambda_{2}^{(G, T)}\right]}\right\}
$$

where:

- $I_{s c}^{(G, T)}$ represents the dependence of $I_{s c}$ vs irradiation $(\mathrm{G})$ and temperature $(\mathrm{T})$.

- $V_{o c}^{(G, T)}$ represents the dependence of $V_{o c}$ vs irradiation and temperature.

- $V$ is the cell output voltage.

- $\lambda_{1}^{(G, T)}$ and $\lambda_{2}^{(G, T)}$ are coefficients with irradiation and temperature dependence. 

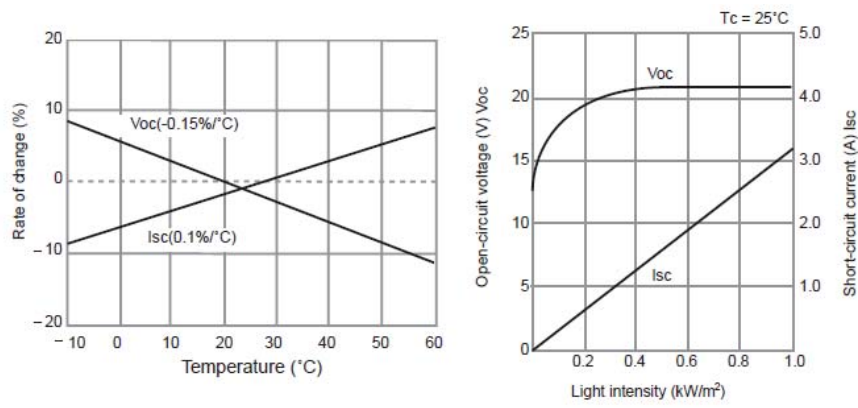

Fig. 4. Example of the dependence of $V_{o c}, I_{s c}$ vs. irradiation and temperature.

\section{A. LPV modelling the voltage at MPP}

The problem considered by MPPT techniques is to automatically find the voltage $V_{M P P}$ or current $I_{M P P}$ at which a PV array should operate to obtain the maximum power output $P_{M P P}$ under a given temperature and irradiance [2].

The MPPT methods can be classified as direct and indirect methods. The direct methods include those methods that use PV voltage and/or current measurements. These methods include the techniques of differentiation, feedback voltage, perturbation and observation (PO), incremental conductance, as well as fuzzy logic and neural network.

The indirect methods are based on the use of a database of parameters that include data of typical $P-V$ curves of PV systems for different irradiances and temperatures, or on the use of mathematical functions obtained from empirical data to estimate the MPP. The indirect methods include the curve fitting, look-up table, open-voltage PV generator and short circuit PV generator. In orden to obtain the $V_{M P P}$ or $I_{M P P}$ curve of a PV generator, the nonlinear characteristic of the PV generator can be off-line modeled, from conventional single diode, two-diode and modified two-diode model, using mathematical equations or numerical approximations [14].

In this work, the STP005S-12/Db solar panel, a 5W PV module, was chosen for modelling. The module has 36 monocrystalline cells serially connected. The key specifications are shown in Table I.

Curve fitting can be used to estimate the parameters on equations (4) and (5) in order to obtain theorical responses of the PV panel.

Figures 5 and 6 depict the response of lineal model given by equation (5) and the response V-I of STP005S-12/Db solar panel at several light intensities and temperatures. The curves $I_{s c}^{(G, T)}, V_{o c}^{(G, T)}, \lambda_{1}^{(G, T)}$ and $\lambda_{2}^{(G, T)}$ can be obtained by interpolation if the range of $\mathrm{T}$ and $\mathrm{G}$ is completed.

The proposed MPPT method is considered as an indirect MPPT method using the curve fitting technique. The output voltage at MPP can be found by simulations as it can be shown in Figure 7 for some irradiances at $25^{\circ} \mathrm{C}$ of temperature and Figure 8, where the output voltage variations at MPP for variations of irradiances in function of temperature are shown071

Considering the fact that the PV modules voltage depends

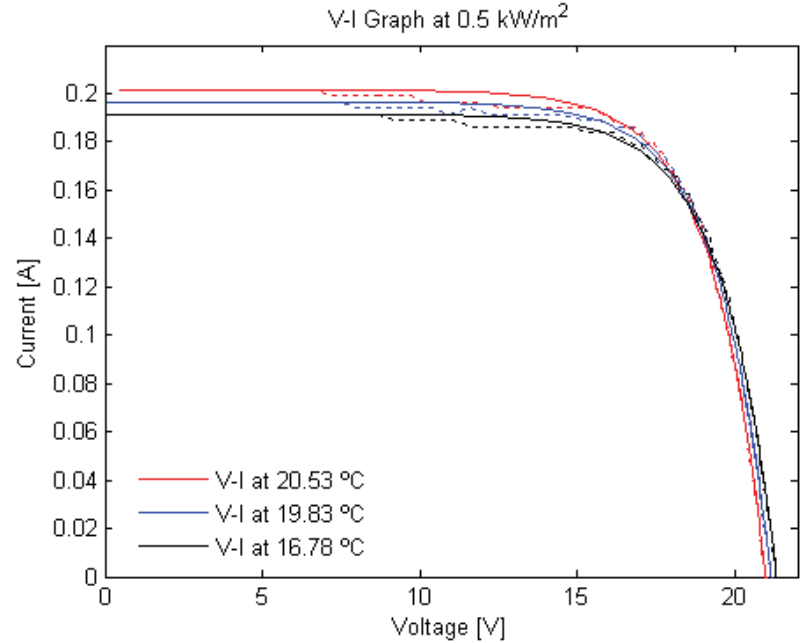

Fig. 5. Response of STP005S-12/Db solar panel at $G=0.5 \mathrm{~kW} / \mathrm{m}^{2}$

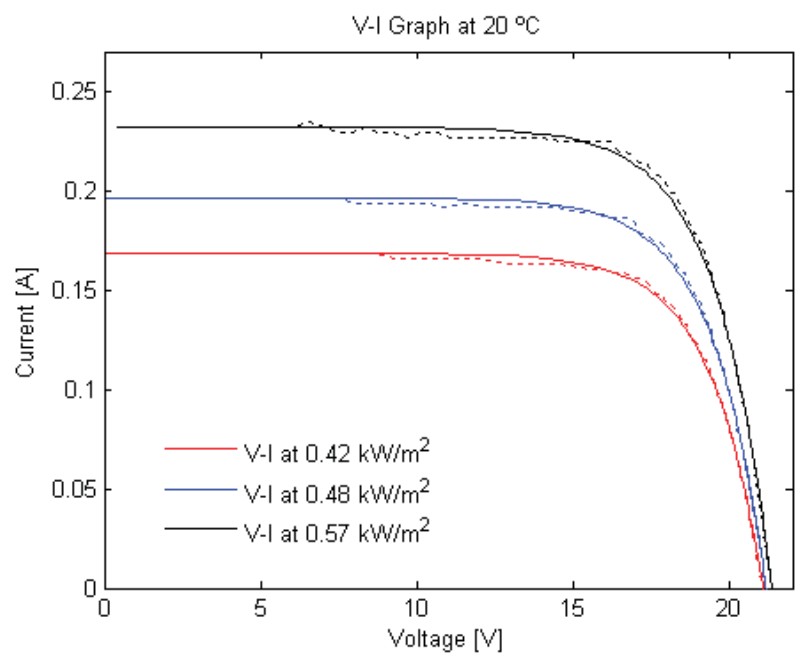

Fig. 6. Response of STP005S-12/Db solar panel at $20^{\circ} \mathrm{C}$

on the cell temperature $\mathrm{T}\left[{ }^{\circ} \mathrm{C}\right]$ and on the sun radiation $\mathrm{G}\left[\mathrm{kW} / \mathrm{m}^{2}\right]$ and by applying the Curve Fitting toolbox of Matlab, in this work the output voltage of Solar Panel Model $\mathrm{STP} 005 \mathrm{~S}-12 / \mathrm{Db}$ at MPP, $V_{m p p}$, is adjusted in function of $\mathrm{T}$ and $\mathrm{G}$ by approximation as defined by the equation:

$$
V_{m p p}(G, T)=p_{1}(G, T) \cdot T+p_{2}(G, T)
$$

TABLE I

Electrical Characteristics OF the Solar PANel Model: STP005S-12/DB.

\begin{tabular}{|c|c|}
\hline Model & STP005S-12/Db \\
\hline Open circuit voltage (Voc) & $21.4[\mathrm{~V}]$ \\
\hline Short circuit current (Isc) & $0.33[\mathrm{~A}]$ \\
\hline Optimun operating voltage (Vmp) & $16.8[\mathrm{~V}]$ \\
\hline Optimum operating current (Imp) & $0.30[\mathrm{~A}]$ \\
\hline Maximun power at STC (Pm) & $5.00[\mathrm{Wp}]$ \\
\hline
\end{tabular}

STC:Irradiance $10^{3} \mathrm{~W} / \mathrm{m}^{2}$, Temperature module $25^{\circ} \mathrm{C}, A M=1.5$. 


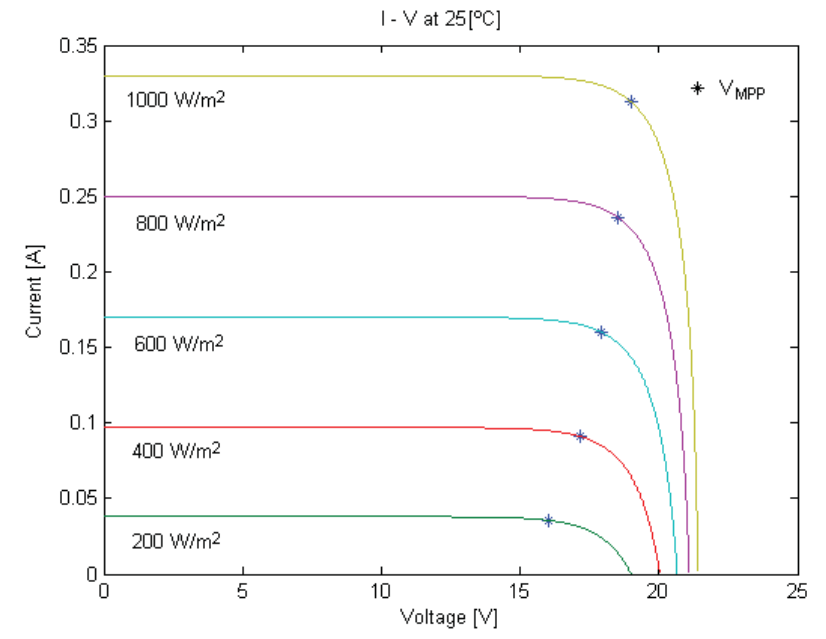

Fig. 7. Simulation of output voltage at MPP of the Solar Panel Model STP005S-12/Db for different irradiances.

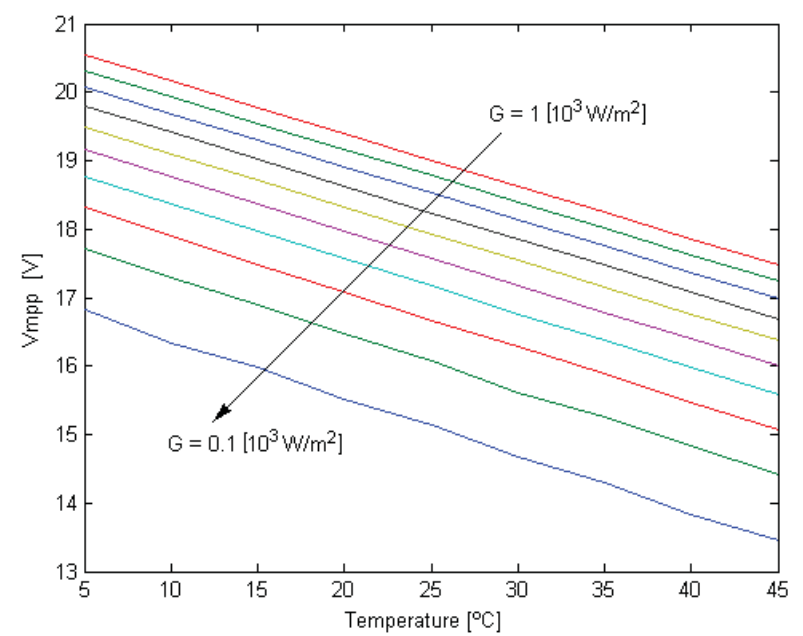

Fig. 8. Variations of output voltage at MPP of the Solar Panel Model STP005S-12/Db.

where:

$$
\begin{aligned}
& p_{1}(G, T)=a_{1}(T) \cdot e^{b_{1}(T) \cdot G}+c_{1}(T) \cdot e^{d_{1}(T) \cdot G} \\
& p_{2}(G, T)=a_{2}(T) \cdot e^{b_{2}(T) \cdot G}+c_{2}(T) \cdot e^{d_{2}(T) \cdot G}
\end{aligned}
$$

and coefficients defined on Table II.

TABLE II

COEFFICIENTS OF EXPRESSIONS $p_{1}(G, T)$ AND $p_{2}(G, T)$.

\begin{tabular}{|c|c|c|}
\hline Coeff. & $p_{1}(G, T)$ & $p_{2}(G, T)$ \\
\hline$a_{i}(T)$ & -0.008455 & 18.740 \\
\hline$b_{i}(T)$ & -3.294000 & 0.1123 \\
\hline$c_{i}(T)$ & -0.078250 & -2.758 \\
\hline$d_{i}(T)$ & -0.027090 & -4.724 \\
\hline
\end{tabular}

\section{State-Space AVERAging Model of CONVERTER BASED ON ULTRACAPACITORS}

Many scientists have worked in order to obtain different capacitor models, Westerlund and Ekstam in [15] proposed that a better capacitor impedance could be

$$
Z(j w)=\frac{1}{(j w)^{\alpha} C} ; 0<\alpha<1
$$

Based on the last expression, the current $i(t)$ across the capacitor is a function of a general voltage $v(t)$

$$
i(t)=C D_{t}^{\alpha} v(t)
$$

It can be noticed that $D_{t}^{\alpha} v(t)$ is the fractional time derivative of the voltage. For different capacitors $\alpha$ is not equal to one, and is close to 0.999. The ultracapacitor can be modeled in intervals where at low frequencies it is similar to a classical capacitor $(\alpha \approx 1)$ and at medium frequencies is characterized by a diffusion effect and is better characterized in the Warburg domain $(j w)^{1 / 2}$ than in the classical Laplace domain $(j w)$ [16]. Figure 9 displays the Nyquist diagram for the capacitor models (real and ideal).

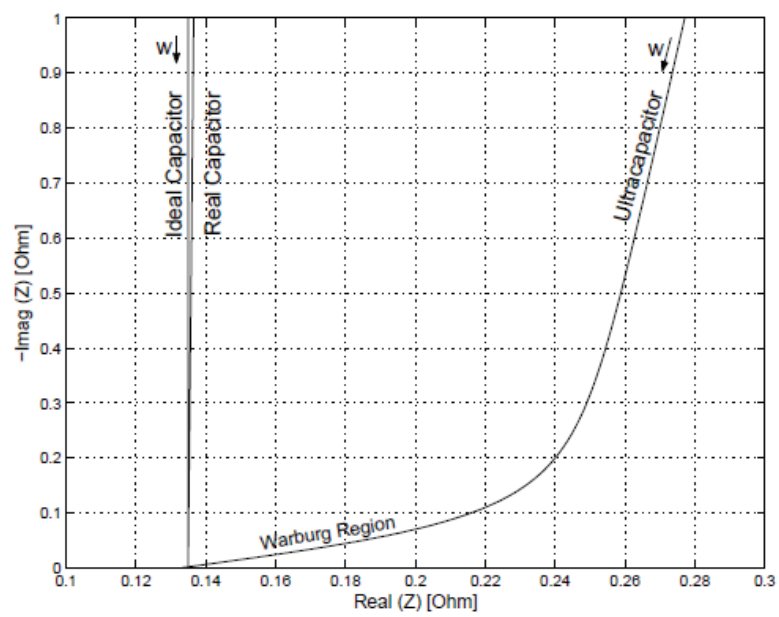

Fig. 9. Nyquist diagram of a capacitor (real and ideal) and an ultracapacitor [16].

Let apply the state-space averaging method to model the converter of Figure 10.

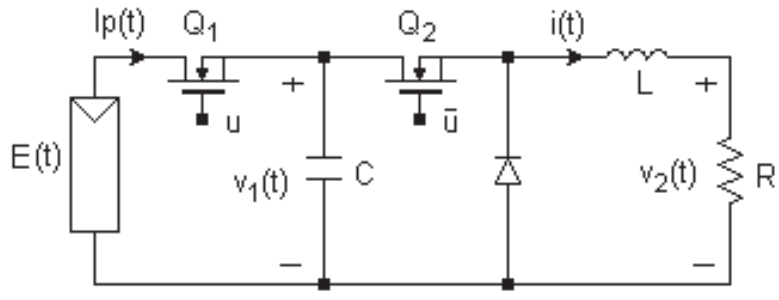

Fig. 10. DC/DC converter system.

1072 The operation of this system is as follows: when $u=1$, the transistor $Q_{1}$ is switched to $\mathrm{ON}$ state (conduction state) 
and the transistor $Q_{2}$ is switched to OFF state, the diode is polarized generating a circuit topology shown in Figure 11 (left side). During this period, the stored energy in the inductor $L$ is transferred to the system load $R$ and the ultracapacitor $C$ is "charging". We say that the circuit is operating in the "charging period".

When the $u=0$ transistor $Q_{1}$ is switched to OFF state, $Q_{2}$ is switched to $\mathrm{ON}$ state and the diode is inversely polarized generating the circuit topology shown in Figure 11 (right side). This second period is known as the "discharging period" due to the fact that the stored energy in the capacitor $C$ is transferred to the system load.

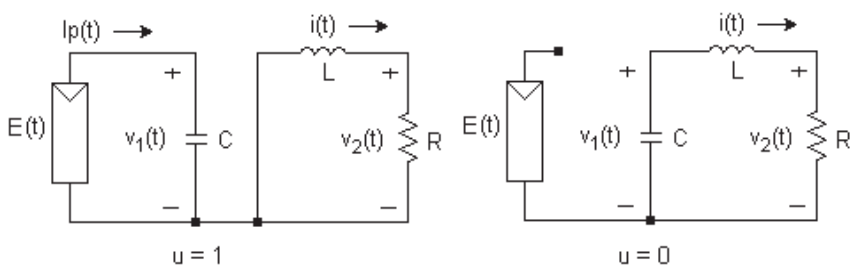

Fig. 11. States of the system. $u=1$ : ON state and $u=0$ : OFF state.

When the Kirchoff and current laws are applied to both circuits topologies of Figure 11, and the obtained models are combined into a single dynamic model, the resulting system of differential equations describing the converter is the following:

$$
\begin{gathered}
C D_{t}^{\alpha} v_{1}(t)=I_{p} u_{a v}-\left(1-u_{a v}\right) i \\
L D_{t}^{1} i(t)=\left(1-u_{a v}\right) v_{1}-i R
\end{gathered}
$$

where $\alpha=0.5$ describes the electrochemical system at medium frequency range and $u_{a v}$ is the average input control.

\section{State Feedback Controller Design}

Let consider the tangent linearization model of the average ideal converter system defined by (7) and around the equilibrium point:

$$
v_{1}^{z}=V_{m p p} ; \quad u_{a v}^{z}=\frac{i}{I_{p}+i}
$$

where $V_{m p p}$ is the normalized reference voltage at MPP.

The linearization of the average model is given by

$$
D_{\tau}^{\alpha} e=u_{a v}^{e}\left(I_{p}+i\right)
$$

where

$$
e=v_{1}-v_{1}^{z}, \quad u_{a v}^{e}=u_{a v}-u_{a v}^{z}
$$

The objective is to find a stabilizing control law $u_{a v}^{e}(t)$ such as:

1) The equilibrium point $e=0$ in equation (8) is locally and asymptotically stable.

2) The control system must reject constant disturbances, like:

$$
\lim _{t \rightarrow \infty}\left[v_{1}-V_{m p p}\right]=0
$$

3) $0 \leq u_{a v}(t) \leq 1, \forall t \geq 0$.
4) The eigenvalues of the average feedback state can be arbitrarily assigned.

An average non-integer and linear state feedback control is sought in the form:

$$
u_{a v}^{e}=-k_{2} I_{\tau}^{0.5} e(t)-k_{1} e(t)
$$

which drives the average stabilization error state $e$ to zero in a generalized exponentially stable fashion. A controller is designed with the help of the average tangent linearization system and it will use, for the average nonlinear system, the control input

$$
u_{a v}=u_{a v}^{e}+u_{a v}^{z}
$$

The equivalent closed loop tangent system is given by

$$
C D_{\tau}^{\alpha}=-\left(I_{p}+i\right) k_{1} e(t)-\left(I_{p}+i\right) k_{2} I_{\tau}^{\alpha} e(t)
$$

and whose equivalent characteristic polynomial can be described as

$$
P(\lambda)=(\lambda+\epsilon)^{2}=\lambda^{2}+a_{1} \lambda+a_{2}
$$

for $a_{1}=\left(I_{p}+i\right) k_{1}$ and $a_{2}=\left(I_{p}+i\right) k_{2}$.

Equating these polynomials to a desired closed loop characteristic polynomial the feedback gains for the rational linear controllers can be obtained [17].

\section{Simulations}

Simulations to assess the effectiveness of the proposed full state feedback controllers have been performed, computed on basis of the tangent linearized systems, to accomplish a stabilization around a normalized equilibrium point value for initial conditions set at origin of coordinates.

The criteria of poles placement to closed loop system is used in order to determinate the feedback gains. In this case, all roots of the characteristic polynomial are defined by $\epsilon$.

The following parameters and design values have been used:

$$
R=10 \Omega, L=0.1 H, C=0.01 F
$$

A slower response to closed loop system, with feedback gain defined by $\epsilon=2$, is proposed because values of $\epsilon>2$ demand faster responses at $t=0^{+}$and the average control input cause a temporary saturation to the corresponding switched controller.

Figures 12 and 13 depict the response of the nonlinear average converter circuit based on ultracapacitors for medium frequencies to control actions of state feedback controller computed on the basis of the linearized tangent average system complemented with the nominal equilibrium control input. Different changes in the operation conditions are considered.

In order to verify that the control system $u_{a v}$ rejects constant disturbances, step signals as changing in sun irradiance at $t=$ $10[s]$, in temperature at $t=15[s]$ and in inductor current at $1073=20[s]$ are used. In Figures 12 and 13 the response of the control system with constant disturbances is shown. 


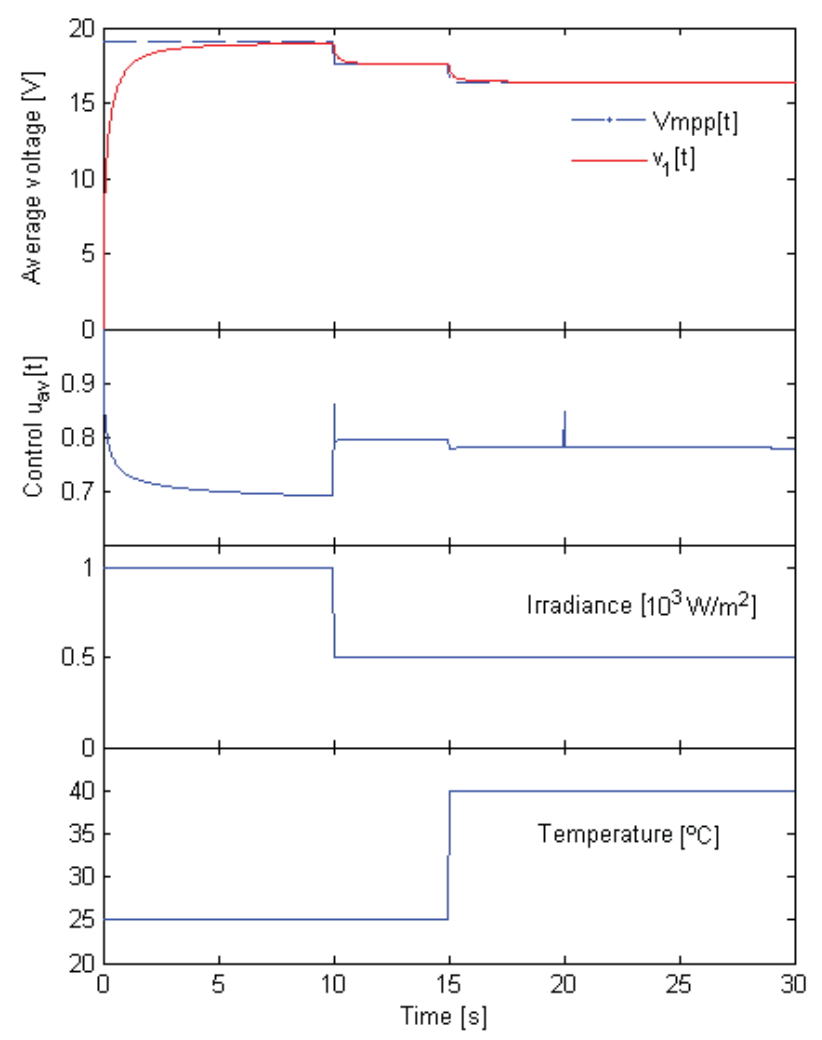

Fig. 12. Response of average converter based on ultracapacitors to linear state feedback controller.

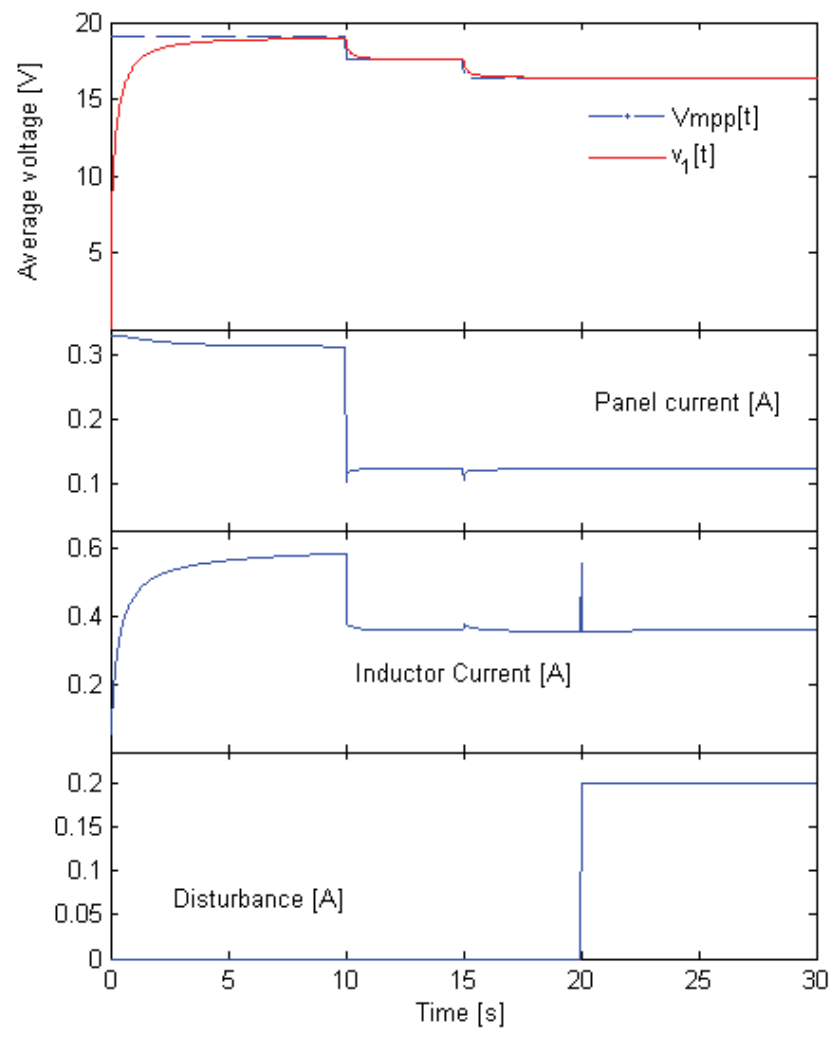

\section{CONCLUSIONS}

In this work, fractional modelling of a DC-DC converter based on ultracapacitors, suitable for many powered electrical systems, is presented. Therefore, a linear feedback controller is designed and verified by simulations results, which represents a strong motivation to the modelling and control of powered electrical systems via fractional control techniques.

\section{ACKNOWLEDGEMENTS}

Authors would like to thank Inter-Ministerial Commission of Spanish Government (DPI2010-17112, MCYT), Catalan Autonomous Government (VIS, Consolidated Research Group) and Cooperation Spanish Agency for International Development (AECID) for funding this research.

\section{REFERENCES}

[1] V. Salas, A. Barrado, A. Lazaro, Review of the maximum power point tracking algorithms for standalone photovoltaic systems, Solar Energy Materials \& Solar Cells, Vol.90, 2006, pp:1555-1578.

[2] Esram, T., and Chapman, Patrick L., Comparison of Photovoltaic Array Maximum Power Point Tracking Techniques, IEEE Transactions on Energy Conversion, Vol. 22, No. 2, June 2007.

[3] R. Kötz, M. Carlen, Principles and applications of electrochemical capacitors, Electrochimica, Acta 45 (2000), 2483-2498.

[4] M. Sullivan, R. Kötz and O. Haas, Electrochemically Modified Glassy Carbon as an Electrochemical Capacitor Material, The Electrochemical Society Proceedings, Vol. 95-29.

[5] Podlubny, I., Fractional Differential equations, San Diego: Academic Press, 1999.

[6] Podlubny, I., Fractional-order systems and $P I^{\lambda} D^{\mu}$-controllers. IEEE Trans. Automatic Cont., vol. 44, no. 1, 1999, pp. 208-214.

[7] J. A. Tenreiro Machado, Analysis and Design of Fractional-Order Digital Control Systems, Journal Systems Analysis-Modelling-Simulation, Gordon \& Breach Science Publishers, vol. 27,pp. 107-122, 1997.

[8] Patrick Lanusse, Alain Oustaloup, Jocelyn Sabatier, Step-By-Step Presentation of a 3rd Generation CRONE Controller Design With An Anti-Windup System, ENOC-2005, EUROMECH Nonlinear Oscillations Conference, Eindhoven, Netherlands, 2005.

[9] Torvik, P.J. and Bagley, R. L., On the Appearance of the Fractional Derivative in the Behavior of Real Materials, Transactions of the ASME, 51, 294-298, 1984

[10] Dzielinski, A. and Sierociuk, D., Stability of Discrete Fractional Order State-Space Systems,Proceedings of the 2nd IFAC Workshop on Fractional Differentiation and its Applications Porto, Portugal, July 19-21, 2006.

[11] Luque, A. and Hegedus,S. Handbook of Photovoltaic Science and Engineering, John Wiley \& Sons Ltd, 2003.

[12] Gow, J.A., Manning, C.D., Development of a photovoltaic array model for use in power-electronics simulation studies, IEE Proc.Electr.Power Appl, vol 146,.n² 2, pp. 193-200, March. 1999.

[13] Goetzberger,A., Hoffmann, V.U., Photovoltaic Solar Energy Generation, Springer, Berlin, 2005.

[14] Hamdy, M.A., A new model for the current-voltage output characteristics of photovoltaic modules, Journal of Power Sources, Vol. 50 No.1-2, 1994, pp: 11-20.

[15] Westerlund, S. and Ekstam, L., Capacitor theory, IEEE Transactions on Dielectrics and Electrical Insulation, vol. 1, 1994.

[16] Quintana, J. J., Ramos, A. and Nuez, I. Identification of the Fractional Impedance of Ultracapacitors, Proceedings of the 2nd IFAC Workshop on Fractional Differentiation and its Applications, Porto, Portugal, 2006.

[17] Martínez, R. and Feliu, V., Linear Control Rational Systems Approach on State Space, Symposium on Applied Fractional Calculus, Badajoz, Spain, 2007.

Fig. 13. Response of average converter to step disturbance. 